\title{
Expansão maxilar em adultos e adolescentes com maturação esquelética avançada
}

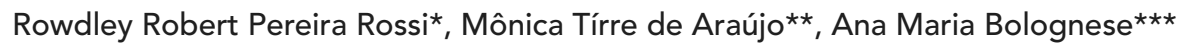

\begin{abstract}
Resumo
A deficiência transversa da maxila é um fator agravante e complicador do tratamento ortodôntico em adultos. Sua correção ainda gera dúvidas e controvérsias entre clínicos e pesquisadores. O objetivo deste trabalho foi analisar e discutir fatores determinantes para o planejamento da expansão maxilar em adultos e adolescentes com maturação esquelética avançada. Ausências dentárias múltiplas, grandes inclinações dentoalveolares para vestibular, recessão gengival, perda óssea alveolar e mobilidade dos dentes posterossuperiores contraindicam a realização de expansão rápida da maxila em indivíduos adultos ou com maturação esquelética avançada. No entanto, esses fatores não devem ser considerados isoladamente para a escolha do método de expansão palatal nesses pacientes. Nessas situações, a Expansão Rápida da Maxila Assistida Cirurgicamente (ERMAC) pode ser uma opção, entretanto, a escolha por essa técnica deve basear-se principalmente na idade do paciente, no grau de maturação esquelética, na localização da deficiência transversa da maxila e nas estruturas anatômicas que oferecem maior resistência à expansão maxilar. O Hyrax é o aparelho mais indicado para indivíduos que vão se submeter à ERMAC.
\end{abstract}

Palavras-chave: Expansão maxilar. Adultos. Deficiência transversa da maxila.

\section{INTRODUÇÃO}

A conscientização sobre a importância da saúde bucal, as exigências estéticas da sociedade e a popularização do tratamento ortodôntico estimularam o paciente adulto a buscar ajuda profissional especializada. Dentre as más oclusões, a deficiência transversa da maxila está entre os mais danosos problemas esqueletais da região craniofacial21. Seu estabelecimento e sua manutenção até a idade adulta produzem um quadro anatomofuncional que torna o tratamento em adultos mais complicado. Sua correção - seja por alterações dentoalveolares, deslocamento transverso dos ossos maxilares auxiliado por osteotomias ou reposicionamento cirúrgico segmentado da base óssea - deve compensar ou restabelecer a relação esquelética transversa normal entre a maxila e a mandíbula. Para alguns autores, o restabelecimento da relação esquelética transversa normal entre as bases ósseas é fundamental para a obtenção de uma oclusão satisfatória e estável ${ }^{18,20}$. Para isso, a sutura palatina mediana (SPM) deve ser aberta e os ossos maxilares separados sem provocar inclinação excessiva dos dentes posterossuperiores ${ }^{13,27}$.

* Metre em Ortodontia pela Universidade do Brasil - UFRJ.

** Professora adjunta de Ortodontia na Universidade do Brasil - UFRJ.

*** Professora titular de Ortodontia na Universidade do Brasil - UFRJ. 
Para outros autores, contudo, a intercuspidação posterior pode ser conseguida por alterações dentoalveolares, sem a necessidade de abertura da SPM $^{14,15}$. Dessa forma, o objetivo desse trabalho foi analisar e discutir fatores determinantes para o planejamento da expansão maxilar em adultos e adolescentes com maturação esquelética avançada.

\section{FUNDAMENTOS TEÓRICOS}

A deficiência transversa dos ossos maxilares se manifesta pela mordida cruzada uni ou bilateral, parcial ou total, além dos casos em que a mordida cruzada não está presente ${ }^{19}$. Comumente, a atresia maxilar é acompanhada do desenvolvimento vertical alveolar excessivo ${ }^{28}$, apinhamento dentário $^{21,28}$, palato profundo e estreito, com largura inferior a $31 \mathrm{~mm}$ (distância intermolares medida no limite cervical) $)^{21,28}$ e contraído na região anterior, além de grandes espaços escuros no corredor bucal, durante o sorriso, caracterizando a síndrome da deficiência maxilar transversa ${ }^{21}$.

Essa displasia pode se apresentar isolada ou associada a outra deformidade esquelética posteroanterior, sendo classificada como real ou relativa, na dependência da dimensão transversa do arco dentário superior e relação posteroanterior das bases ósseas. A condição esquelética de Classe II, frequentemente, é acompanhada de constrições nas dimensões transversas do arco superior, conferindo à maxila forma triangular e atrésica bem característica. No entanto, a discrepância basal posteroanterior de Classe II esconde o envolvimento transversal da maxila, devido à oclusão mais posterior do arco inferior. Na discrepância basal posteroanterior de Classe III, a posição do arco dentário inferior à frente do superior acentua a atresia maxilar existente ou projeta uma atresia inexistente ${ }^{18,21}$. Dessa forma, o diagnóstico diferencial entre deficiência transversa real ou relativa do arco maxilar pode ser feito pela análise dinâmica dos modelos de gesso em relação de chave de oclusão. A persistência da mordida cruzada posterior na posição dentária de chave de oclusão revela deficiência transversa real do arco superior ${ }^{18}$. Em pacientes com padrão esquelético Classe I, se manifesta por meio da mordida cruzada posterior unilateral, apesar do caráter simétrico da atresia do arco superior, ou pela atresia simultânea dos arcos superior e inferior. Naquelas, a manifestação unilateral explica-se pelo deslocamento funcional mandibular. Nos casos em que a atresia da maxila não é visível, devido à atresia simultânea do arco inferior, os dentes posteroinferiores, frequentemente, estão contraídos e com inclinação lingual exagerada, e os dentes posterossuperiores apresentam grande inclinação bucal ${ }^{13,19,21}$. Nesses casos, a oclusão posterior é aparentemente normal, mas, em exame minucioso, verifica-se a maxila atrésica, na qual as cúspides palatinas dos dentes posterossuperiores estão inclinadas para vestibular, abaixo do plano oclusal, acentuando a curva de Wilson e, frequentemente, causando interferências oclusais durante as funções ${ }^{21}$.

O tratamento da Deficiência Transversa Maxilar por meio da Expansão Rápida dos Ossos Maxilares (ERM) é conhecido há mais de 140 anos, mas seus aspectos clínicos, radiográficos e mecanismos de ação foram melhor definidos e difundidos após estudos realizados pelo Dr. Andrew J. Haas, na década de 60. A partir de então, inúmeras investigações clínicas e experimentais foram relatadas na literatura e a ERM tornou-se um método rotineiramente usado em pacientes em crescimento ${ }^{11,19}$.

Em adultos, a ERM possui limitações e complicações, como a resistência à expansão, ausência ou pequena abertura da SPM, predominância de expansão dentoalveolar em relação ao ganho transverso da base óssea ${ }^{2,13,15,17}$, excessiva inclinação vestibular e extrusão dos dentes posterossuperiores, absorção da cortical óssea vestibular, recessão gengival, dor, edema, ulcerações e isquemia da mucosa palatal, além de elevado grau de recidiva ${ }^{2}$.

É consenso na literatura que a idade e a maturação esquelética avançada tornam o prog-

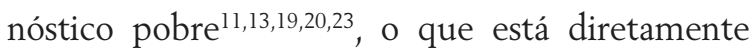
relacionada ao grau do efeito ortopédico ${ }^{9,10,11,17}$. 
Em idades precoces, durante o período das dentaduras decídua, mista e permanente jovem, esse tratamento possui grande efeito ortopédico, quando comparado à dentadura permanente adulta, pois a resposta sutural e esquelética é mais favorável durante o período de crescimento ${ }^{2,11,23}$. Em adultos, o aumento da dimensão esquelética transversa do palato promovido pela ERM é peque$\mathrm{no}^{2,15,17}$, sendo predominantemente dentoalveolar. Assim, a separação entre os incisivos centrais, sinal clínico de abertura da SPM, raramente ocorre ${ }^{14,15}$. Portanto, para muitos autores, o período durante o surto de crescimento ou até os 15 anos de idade é considerado ideal para a realização da expansão rápida da maxila ${ }^{11,19,23}$.

O crescimento transverso do palato pela atividade osteogênica da sutura palatina mediana continua até a idade de 16 anos nas meninas e 18 anos nos meninos ${ }^{23}$. No entanto, o grau de sinostose da sutura palatina mediana possui grande variabilidade individual. Pearson e Thilander ${ }^{26}$, ao avaliarem material de autópsias humanas, encontraram um indivíduo de 27 anos sem nenhum grau de obliteração da sutura palatina mediana. Entretanto, sabe-se que existe relação entre o aumento da maturação esquelética e a obliteração da sutura palatina mediana ${ }^{29}$. Por isso, historicamente, a interdigitação da sutura palatina mediana foi responsabilizada pela resistência à expansão palata1 ${ }^{23,26}$. Entretanto, estudos têm demonstrado que a maior resistência à ERM é representada pelo aumento da ma-

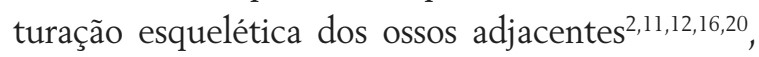
especialmente o osso zigomático ${ }^{2,11,12,20}$ - pela maior interdigitação de algumas suturas craniofaciais $^{2,8,12,16,20,27}$, principalmente as temporozigomática, frontozigomática, zigomatomaxilar 2,8,12,16,20,27 - e o osso esfenoide, pelo aumento da interdigitação da sutura pterigomaxilar $2,16,18,25$.

A determinação do grau de maturação esquelética - por meio da análise das radiografias de punho e mão - é de grande importância no diagnóstico e plano de tratamento das más oclusões esqueléticas, pois, dentre as idades biológicas (idades cronológica, esquelética, morfológica, dentária e circumpuberal), a idade esquelética representa com mais fidelidade o desenvolvimento somático geral do indivíduo ${ }^{30}$.

A resistência do esqueleto craniofacial à expansão palatal e abertura da sutura palatina mediana, em pacientes com maturação esquelética avançada, estimulou o surgimento de vários protocolos de osteotomias maxilares como adjuntas da expansão (ERMAC - Expansão Rápida da Maxila Assistida Cirurgicamente) - com a finalidade de diminuir essa resistência ${ }^{1,2,5,8,20,24,26,27,28}$ separando o osso basal maxilar dos seus principais suportes do crânio ${ }^{18}$, de maneira segura, simples e confiável, para obter aumento permanente da largura maxilar ${ }^{8,27}$, com inexpressiva inclinação dentária ${ }^{2}$.

Basicamente, dois procedimentos cirúrgicos são amplamente relatados na literatura para a correção da deficiência transversa dos ossos maxilares em pacientes adultos: (1) osteotomia maxilar Le Fort I segmentada (Expansão Cirúrgica Maxilar Segmentada - ECMS), com o objetivo de liberar a maxila dos ossos contíguos e segmentála, para promover o reposicionamento lateral das partes e a correção da atresia maxilar durante o ato cirúrgico ${ }^{6}$; e (2) osteotomia maxilar parcial (ERMAC), para reduzir a resistência à expansão, realizada em conjunto com um dispositivo expan$\operatorname{sor}^{1,2,8,20,23,25,27,28}$. A escolha da alternativa cirúrgica mais adequada deve considerar o grau de morbidade, a quantidade de expansão planejada e a necessidade de cirurgia ortognática após a resolução do problema esquelético transverso. Indivíduos com comprometimento esquelético nos planos posteroanterior e/ou vertical, combinado com deficiência transversa da maxila, podem submeter-se a um único momento cirúrgico. No entanto, o tratamento em dois estágios (primeiro a ERMAC e depois a cirurgia posteroanterior e/ou vertical) permite maiores expansões ${ }^{22}$, com grande estabilidade ${ }^{28}$, e elimina a necessidade de correção do problema transverso no momento da correção cirúrgica sagital e/ou vertical, o que simplifica a cirurgia e torna 
a osteotomia maxilar unissegmentar, e ainda reduz as chances de complicações, como a lesão de vasos importantes e a necrose dos tecidos. Clinicamente, o resultado transverso da ERMAC é mais estável do que o da expansão cirúrgica maxilar segmenta$\mathrm{da}^{3,26}$. Phillips et al. (1992 apud BYLOFF, MOS$\mathrm{SAZ}^{5}$ ) encontraram alto grau de recidiva (40\%) quando a Le Fort I segmentada era selecionada para a correção de problemas transversos.

A manutenção dos resultados alcançados ao longo do tempo constitui um grande desafio clínico frente à ERM em adultos. Indivíduos em crescimento possuem maior estabilidade quando comparados aos adultos e adolescentes com maturação esquelética avançada ${ }^{10}$. Nesses, a grande inclinação lateral dentoalveolar decorrente da ERM torna a recidiva imprevisível e sem controle, e os dentes posterossuperiores tendem a produzir oclusão topo a topo ${ }^{7}$, pois a musculatura peribucal raramente permite a estabilização dessa inclinação dentoalveolar ${ }^{8}$. Wertz ${ }^{31}$ relatou $63 \%$ de recidiva após a ERM em pacientes com mais de 18 anos, discordando dos achados de Handelman, Wang, BeGole e Haas ${ }^{15}$, nos quais a porcentagem de recidiva foi de 8 a $9 \%$, sem a recorrência da mordida cruzada, após 5,9 anos de contenção, em média. Em relação à estabilidade dos resultados obtidos com a ERMAC, Bell e Epker² não relataram recidiva nos 15 casos tratados, após 19 meses da cirurgia. Glassman, Nahigian, Medway et al. ${ }^{8}$ tiveram sucesso clínico em todos os casos tratados, sem recidiva, após cinco anos do tratamento. Resultado confirmado pelos estudos de Pogrel, Kaban, Vargervik e Baumrind ${ }^{27}$, que comprovaram que a expansão palatal permaneceu estável por 12 meses, com recidiva média de $0,88 \pm 0,48 \mathrm{~mm}$.

\section{DISCUSSÃO}

A maxila atrésica possui várias características associadas que lhe são peculiares. Por isso, o clínico deve observar, minuciosamente: a condição transversa por meio de exame clínico, análise dos modelos de gesso e radiografia cefalométrica posteroanterior, considerando a morfologia do palato (atresia e profundidade) e dos processos alveolares (inclinação e desenvolvimento vertical); a relação espacial transversa e posteroanterior da maxila em relação à mandíbula (mordida cruzada real ou relativa); o grau de compensação dentária dos dentes posteriores no sentido transverso; a análise do sorriso (espaços escuros no corredor bucal) e o grau de discrepância transversa esquelética e dentoalveolar.

O tratamento da deficiência transversa da maxila em adultos e adolescentes com maturação esquelética avançada é controverso e suscita muitas dúvidas. Entretanto, a decisão de qual abordagem utilizar, nas mais diferentes situações de atresia maxilar, está na dependência de alguns fatores, sendo que nenhum deles deve ser analisado de forma isolada.

A idade e a maturação esquelética do paciente são importantes fatores a serem considerados durante o plano de tratamento da deficiência maxilar. Após o Surto de Crescimento Puberal, o prognóstico da abertura da SPM e do ganho esquelético transverso do palato por meio da ERM não é favorável, pois existe relação direta entre o aumento da resistência esquelética à expansão e o aumento da idade do paciente ${ }^{1}$. Analisando esse aspecto, Glassman, Nahigian, Medway et al. ${ }^{8}$ realizaram osteotomias maxilares como adjuntas no tratamento da atresia maxilar em indivíduos com idades que variaram dos 14 aos 44 anos, Bell e Epker $^{2}$ em indivíduos de 15 a 27 anos, e Byloff e Mossaz $^{5}$ em pacientes de 18 a 41 anos.

Reconhecer a severidade da deficiência transversa da maxila e identificar os componentes dentoalveolares e esqueléticos envolvidos ajuda o profissional a definir melhor o problema. Em adultos, Capelozza Filho et al. ${ }^{6}$ conseguiram pequeno ganho transverso, após a ERM, de 3,26mm e 1,46mm nas distâncias intermolares e intercaninos, respectivamente. Handelman, Wang, BeGole e Haas ${ }^{15}$ encontraram valores superiores, em média de 5,9mm de expansão dentária na região 
dos primeiros molares e de $2,8 \mathrm{~mm}$ na região dos caninos. Mas vale ressaltar que $80 \%$ dessas alterações foram dentoalveolares e a expansão foi realizada até a instalação de mordida cruzada bucal superior. Utilizando a Expansão Semi-Rápida da Maxila (ESRM) em adolescentes com maturação esquelética avançada, Iseri e Ozsoy ${ }^{17}$ conseguiram $2,47 \mathrm{~mm}$ de expansão esquelética e $7,36 \mathrm{~mm}$ de ganho transverso na região dos molares, com menores efeitos dentoalveolares (64\%) quando comparados com estudos em adultos ${ }^{14,15}$ e mais próximo dos valores encontrados em crianças submetidas à ERM, nos quais $56 \%$ da expansão foram decorrentes de deslocamento transverso dos ossos maxilares ${ }^{15}$. A natureza da correção da deficiência transversa dos ossos maxilares - seja por expansão dentoalveolar associada à inclinação dentária ${ }^{6,14,15,17}$, seja por deslocamento lateral da base óssea - deve ser levada em consideração no planejamento ortodôntico.

Em adultos com discrepância transversa suave (até $4 \mathrm{~mm}$ ), medida clinicamente na região dos molares, a correção pode ser conseguida por compensação dentária, por meio da qual os arcos são coordenados para promover $1 \mathrm{~mm}$ de expansão nos segmentos posterossuperiores e $1 \mathrm{~mm}$ de contração nos posteroinferiores, de cada lado ${ }^{3,22}$. Mas, segundo $\mathrm{Haas}^{13}$, a língua raramente permite a estabilidade da contração dentária inferior. A ERMAC frequentemente é indicada para discrepâncias transversas entre a maxila e a mandibula acima de $5 \mathrm{~mm}^{1,3,27}$. Medeiros e Medeiros ${ }^{22}$ recomendam a expansão cirúrgica maxilar segmentada para ganhos transversos de até $7 \mathrm{~mm}$, quando também é necessário resolver cirurgicamente o problema esquelético vertical e/ou posteroanterior. Expansão esquelética transversa acima de $7 \mathrm{~mm}$ deve ser obtida por meio da ERMAC ${ }^{22}$. A relação entre o grau de severidade da discrepância esquelética transversa entre a maxila e mandíbula e o limite para a indicação do paciente adulto à cirurgia ainda não são um consenso. Dessa forma, levando-se em consideração apenas a gravidade da má oclusão, torna-se prudente indicar a ERMAC, em indivíduos com maturação esquelética avançada, para discrepâncias transversas severas (maiores do que $7 \mathrm{~mm}$ ), onde o aumento da largura da base óssea maxilar se faz necessário para a obtenção de uma oclusão satisfatória (Fig. 1, 2).

De acordo com Betts, Vanarsdall e Barber et al. ${ }^{3}$, a expansão rápida da maxila deve ser limitada às discrepâncias suaves (menores ou iguais a $4 \mathrm{~mm}$ ) e moderadas ( 5 a $6 \mathrm{~mm}$ ), devido ao grande componente dentoalveolar desses tratamentos. Outro estudo indica que as mudanças transversas, esqueléticas e dentárias são mais estáveis com a expansão lenta da maxila imediatamente após a abertura da SPM (ESRM - Expansão Semi-Rápida da Maxila), o que sugere que esse protocolo produz menor resistência às estruturas nasomaxilares e maior estímulo para o processo de adaptação das estruturas circunvizinhas ${ }^{17}$.

Embora em adultos, após a expansão palatal, o ganho esquelético transverso seja relativamente pequeno, a expansão alveolar pode ser uma alternativa para aumentar a largura do palato e promover a intercuspidação posterior ao final do tratamento ortodôntico corretivo (Fig. 3), sem, entretanto, promover a abertura da SPM, radiograficamente analizada ${ }^{14,15}$.

A inclinação dentoalveolar posterossuperior deve ser cuidadosamente observada durante o planejamento da expansão palatal ${ }^{13}$, principalmente em adultos. Handelman ${ }^{14}$ encontrou acentuado aumento na inclinação vestibular nos primeiros molares superiores, de $5^{\circ}$ a $9^{\circ}$, após a ERM. Comparando o grau de inclinação dos molares superiores e dos processos palatinos de crianças e adultos submetidos à ERM e a tratamento ortodôntico corretivo fixo com sistema Edgewise standard, Handelman, Wang, BeGole e Haas ${ }^{15}$ constataram, nos adultos, valores próximos a 3,1 $\pm 5,7^{\circ}$ e $4 \pm 3,9^{\circ}$ de cada lado na inclinação vestibular dos primeiros molares e processos dentoalveolares, respectivamente. Nas crianças, nenhuma alteração na angulação dos primeiros molares e processos palatinos da maxila foi observada. 

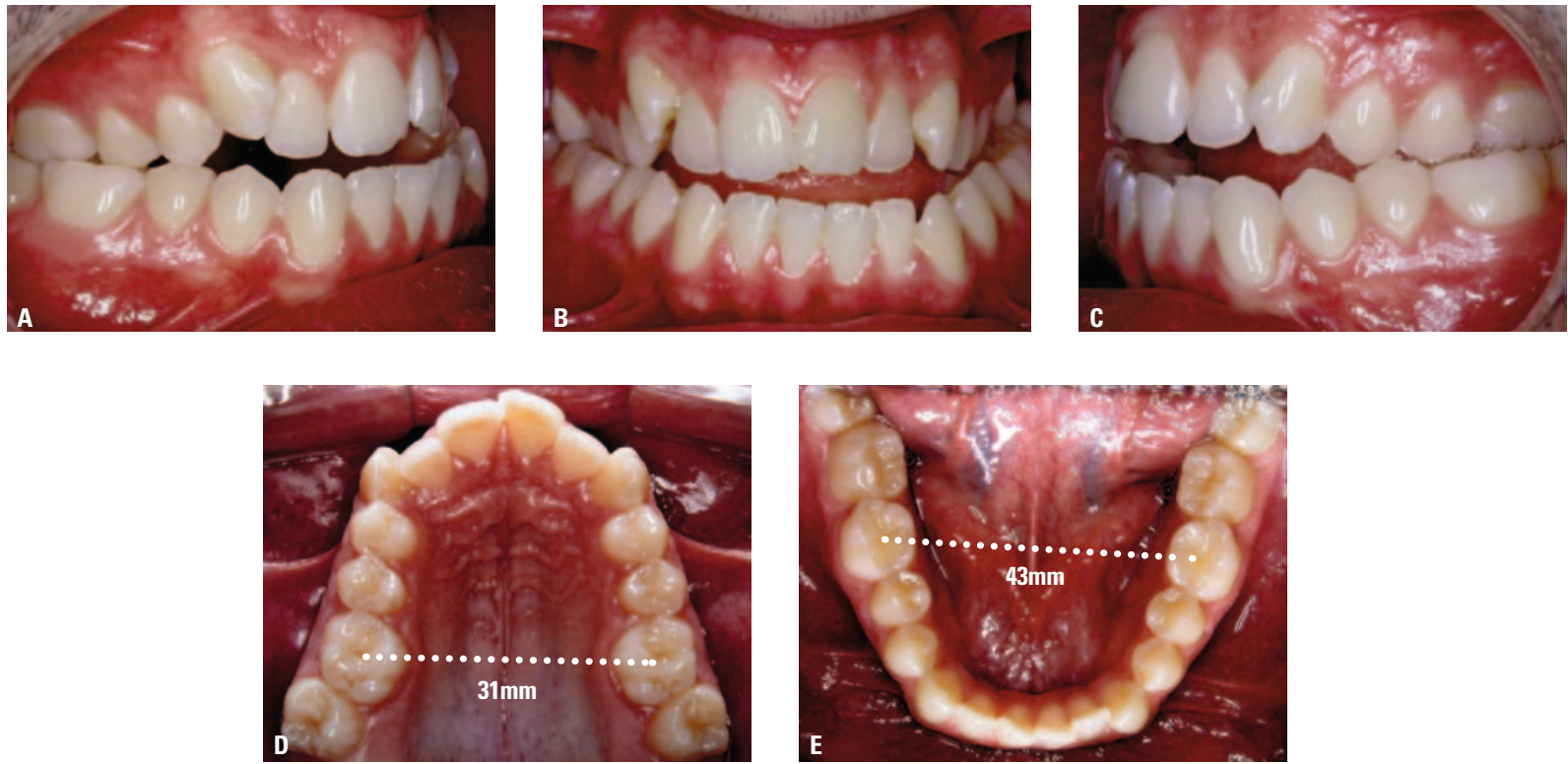

FIGURA 1 - Diagnóstico clínico da discrepância transversa entre a maxila e a mandíbula. As fotografias ilustram o caso de um paciente do gênero masculino, com 18 anos de idade, palato profundo e atrésico e discrepância transversa severa entre a maxila e a mandíbula (12mm), medida clinicamente. Apresentava também mordida cruzada posterior e mordida aberta anterior, relação molar bilateral de Classe III e inclinação lingual exagerada dos dentes posteroinferiores.
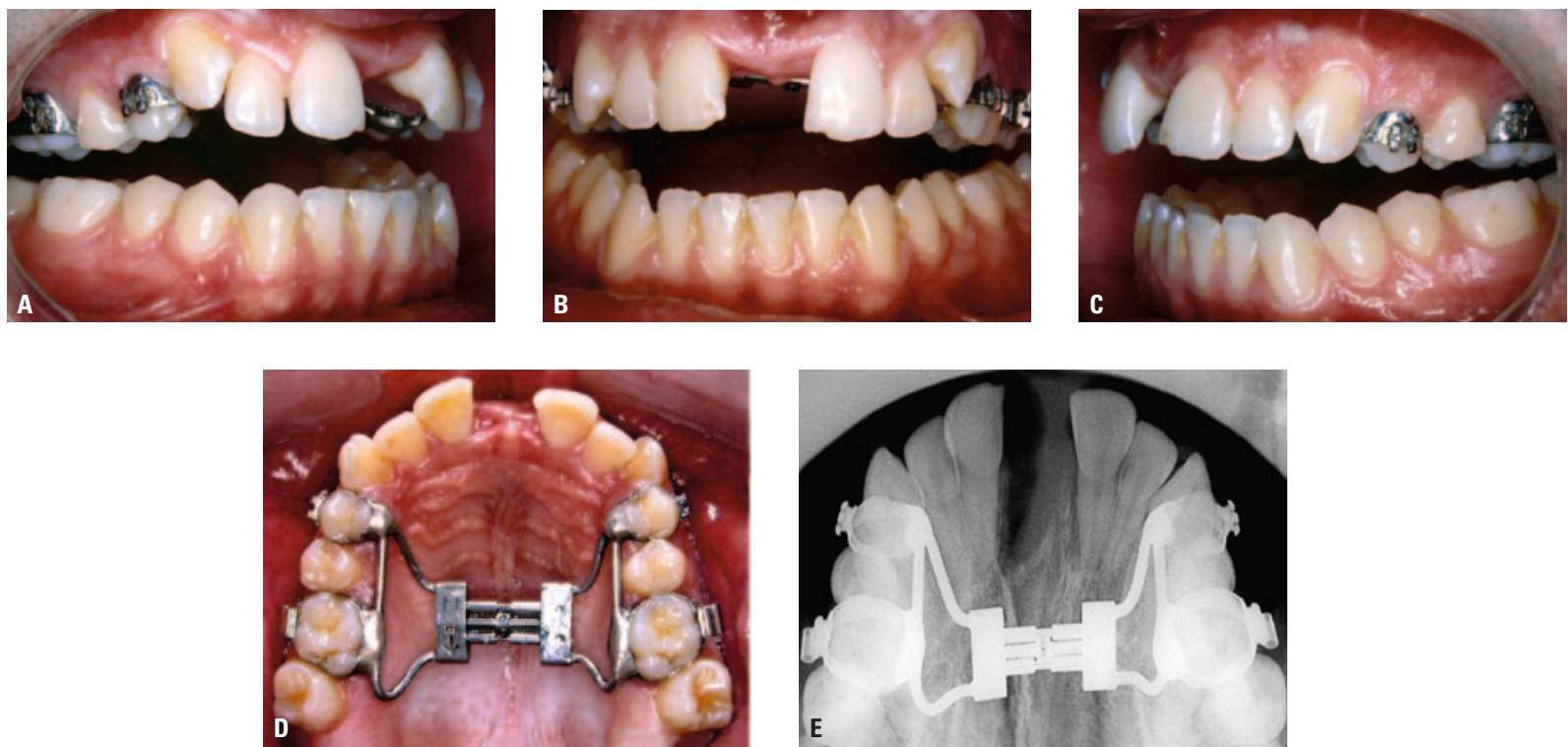

FIGURA 2 - Resultado clínico e radiográfico após a ERMAC do paciente apresentado na figura 1. A, B, C) Fotografias intrabucais lateral direita, frontal e lateral esquerda ilustrando um grande diastema interincisivos, abertura temporária da mordida (contato prematuro entre os segundos molares superiores e inferiores) e giro horário da mandíbula. Os dentes se encontram escurecidos devido ao uso de colutório de clorexidina a 0,12\% no pós-operatório. D) Fotografia intrabucal oclusal mostrando o aumento na largura do palato e do comprimento do arco superior. E) Radiografia oclusal da maxila ilustrando a abertura da sutura palatina mediana. 

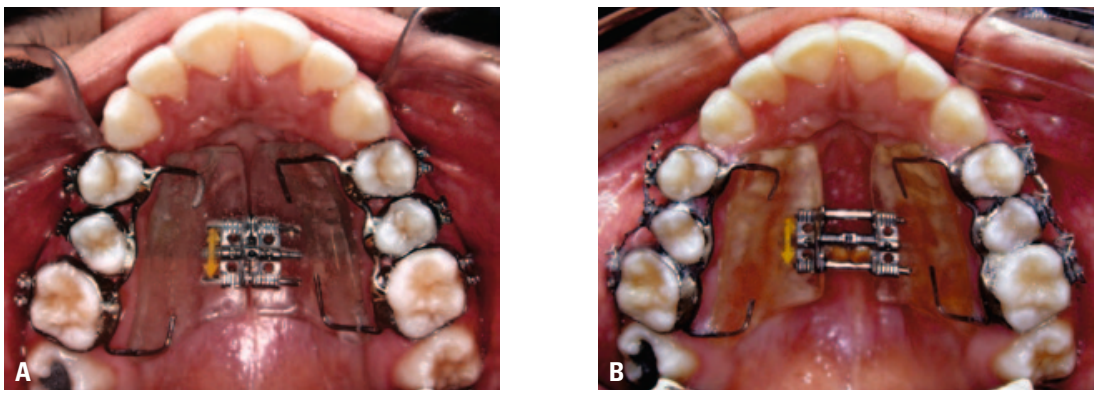

FIGURA 3 - Fotografias intrabucais oclusais superiores mostrando o aspecto clínico transverso: A) antes e B) após a ERM. Paciente do gênero feminino com 18 anos de idade: ficam evidentes, principalmente, as alterações transversas dentoalveolares.

Grandes inclinações dentoalveolares posterossuperiores para vestibular contraindicam a utilização da ERM e a coordenação dos arcos (expansão superior), principalmente quando essa discrepância for severa. Haas ${ }^{13}$ afirmou que é impossível manter essa inclinação, sem contenção, por toda a vida. Dessa forma, métodos que realmente promovam ganho esquelético transverso e menor inclinação dentoalveolar vestibular devem ter preferência nesses casos. Entretanto, em casos de discrepância transversa de suave a moderada, com pouca ou nenhuma inclinação vestibular dos processos alveolares, a ERM e/ou a ESRM podem ser realizadas, pois grande parte da expansão observada se deve à inclinação e ao remodelamento do processo alveolar, do terço apical à região média do palato (Expansão Alveolar Alta) ${ }^{14}$.

A quantidade e a qualidade da ancoragem ortodôntico-ortopédica para os aparelhos expansores devem ser consideradas na determinação do método para correção da deficiência transversa. Ausências dentárias posterossuperiores múltiplas, recessão gengival avançada ${ }^{14,15}$ e perda óssea alveolar $^{9}$ comprometem a eficácia dessa ancoragem. Sabe-se que a recessão gengival e a perda óssea alveolar aumentam com a idade e com a utilização de métodos de expansão que promovem extrusão dentária e inclinação dentoalveolar para vestibular exagerada. Dessa forma, aumenta a necessidade de osteotomias maxilares para reduzir a resistência à expansão em indivíduos com maturação esquelética avançada que apresentam periodonto de sustentação abalado ${ }^{1,9,14,15}$ e ausências dentárias posterossuperiores (Fig. 4). As osteotomias liberam parcialmente a maxila do crânio, e o deslocamento lateral dos processos palatinos acontece facilmente, com menor pressão sobre os dentes de ancoragem, proporcionando menor extrusão e inclinação vestibular dos mesmos. Segundo Haas ${ }^{9}$, a ERMAC está indicada quando existir 30\% ou mais de perda óssea nos dentes de suporte do aparelho expansor.

O Hyrax (dentossuportado) é o aparelho de escolha nos casos de ERMAC, pois facilita a higienização, não provoca lesões ulcerativas e eritomatosas na mucosa palatina ${ }^{4,22}$, além de não comprometer a vascularização dos ossos maxilares ${ }^{22}$. Contudo, em casos de deficiência maxilar severa associada à recessão gengival avançada, perda óssea alveolar e/ou ausências dentárias posterossuperiores, o aparelho tipo Haas ${ }^{10-13}$ é indicado para adultos que irão se submeter à ERMAC. O bloco de resina acrílica que recobre o palato duro oferece ancoragem dentomucossuportada, o que diminui as forças sobre os dentes de ancoragem e proporciona maior compressão e dissipação das forças de ativação do parafuso expansor para as bases ósseas apicais, favorecendo a expansão palatal e diminuindo os efeitos deletérios sobre o periodonto e o processo alveolar dos dentes posterossuperiores. A higienização tem que ser reforçada e o protocolo de ativação deve ser lento, para que o bloco de 

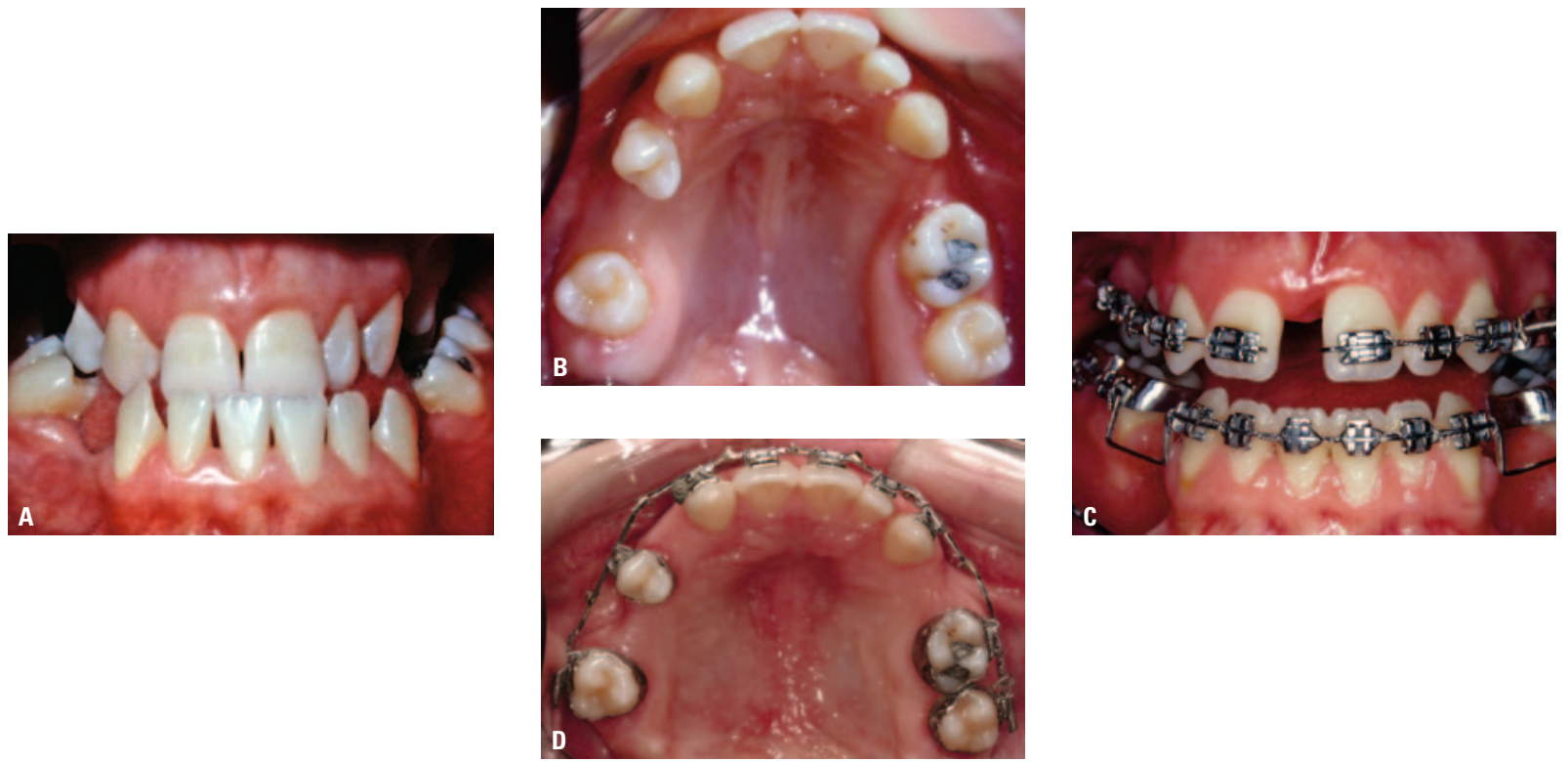

FIGURA 4 - Aspecto clínico transverso antes e após a ERMAC utilizando aparelho expansor tipo Haas. Paciente com 19 anos de idade, do gênero feminino, com perdas dentárias múltiplas e periodonto de sustentação abalado. A) Fotografia intrabucal frontal mostrando severa discrepância transversa entre a maxila e a mandíbula, mordida cruzada total e grande inclinação lingual dos dentes posteroinferiores. B) Fotografia intrabucal oclusal superior ilustrando a severa deficiência transversa da maxila e ausências dentárias (12, 15, 16, 17, 24, 25 e 26). C) Após a ERMAC, com grande diastema interincisal, melhora na relação transversa entre a maxila e a mandíbula, abertura temporária da mordida (contato prematuro devido à sobrecorreção) e giro horário da mandíbula. D) Após o tratamento corretivo fixo, mostrando grande aumento na largura do palato e no comprimento do arco superior.

acrílico do aparelho expansor respeite a integridade dos tecidos moles do palato (Fig. 4).

Muitas complicações da expansão palatal convencional podem ser eliminadas pela ERMAC, realizada com auxílio de vários tipos de osteotomias maxilares ${ }^{1,2,5,20,24,25,27,28}$. A escolha da técnica cirúrgica mais adequada deve se basear, principalmente, no grau de maturação esquelética, na idade do paciente e nas estruturas anatômicas que oferecem maior resistência à expansão maxilar: crista nasomaxilar (suporte anterior), crista zigomatomaxilar (suporte lateral), sutura pterigomaxilar (suporte posterior) e sutura palatina mediana (suporte medial).

Vários protocolos de osteotomias maxilares são descritos na literatura, incluindo ou não todas as estruturas citadas acima. A osteotomia lateral se faz necessária em todos os procedimentos cirúrgicos da ERMAC, pois libera a maxila da crista zigomatomaxilar e das estruturas craniofaciais ligadas a essa, relatada na literatura como a principal região de resistência à expansão palatal ${ }^{2,11,12}$. A realização de osteotomia unilateral e a utilização do lado não-operado como ancoragem são indicadas nos casos de mordida cruzada esquelética unilateral ${ }^{2,20,24}$. Entretanto, o caráter simétrico da deficiência maxilar contraindica essa abordagem. A realização apenas da osteotomia medial pode resultar em maior inclinação bucal dos dentes posterossuperiores, maior recidiva e menor expansão na região dos caninos ${ }^{5}$. A osteotomia do suporte anterior e a abertura da SPM, com auxílio de osteótomo, provavelmente possibilitem maior ganho transverso anterior ${ }^{1}$. A osteotomia mediopalatal, bilateralmente à SPM, deve ser considerada em casos de alterações morfológicas (torus palatino $)^{2}$. De acordo com alguns autores, a liberação dos processos pterigóideos se faz necessária, pois, diferentemente da maxila - que é composta por dois ossos -, o esfenoide é único ${ }^{1,3}$. Para Bell e Epker $^{2}$, esse procedimento cirúrgico deve ser indicado, seletivamente, quando houver necessidade 
significativa da expansão intermolares. Quando a extração dos terceiros molares superiores estiver indicada, a osteotomia vertical no sítio da extração é uma opção para aumentar o ganho transverso na região posterior do arco. No entanto, frequentemente, o maior grau de deficiência transversa da maxila está concentrado na região média e/ou anterior do arco superior, o que, muitas vezes, torna a liberação da sutura pterigomaxilar desnecessária, permitindo que o procedimento seja menos invasivo, menos cruento, com menores complicações pós-operatórias, além da maior possibilidade de realizá-lo em ambiente ambulatorial.

\section{CONCLUSÃO}

Após análise e discussão da bibliografia utilizada, é possível concluir-se que:

1) Ausências dentárias múltiplas, grandes inclinações dentoalveolares para vestibular, recessão gengival, perda óssea alveolar e mobilidade dos dentes posterossuperiores contraindicam a realização da expansão rápida da maxila em indivíduos adultos ou com maturação esquelética avançada. Entretanto, esses fatores não devem ser considerados isoladamente para a escolha do método de expansão palatal em adultos.

2) A literatura científica não mostra consenso em relação à idade ideal para a indicação da ERMAC, porém, essa está indicada em discrepâncias transversas severas e em indivíduos adultos ou com maturação esquelética avançada.

3) A escolha da técnica cirúrgica (ERMAC) deve basear-se principalmente na idade do paciente, no grau de maturação esquelética, nas estruturas anatômicas que oferecem maior resistência à expansão maxilar e na localização da atresia palatal.

4) O Hyrax é o aparelho expansor de escolha para indivíduos que irão se submeter à ERMAC. Entretanto, em casos de deficiência maxilar severa associada a avançada recessão gengival, perda óssea alveolar, mobilidade e ausências dentárias posterossuperiores, o aparelho tipo Haas (dentomucossuportado) está indicado.

Enviado em: abril de 2007 Revisado e aceito: junho de 2009

\title{
Maxillary expansion in adults and adolescents with advanced skeletal maturation
}

\begin{abstract}
The transverse maxillary deficiency is a complication factor in adults' orthodontic treatment. Its correction still generates doubts and controversies between clinicians and researchers. The aim of this paper is to discuss the determinative factors in planning maxillary expansion in adults and adolescents with advanced skeletal maturation. Multiple dental absences, several dentoalveolar buccal inclination, gingival recession, alveolar bone loss and mobility of posterosuperior teeth contraindicate the rapid maxillary expansion in adults or patients with advanced skeletal maturation. However, these factors should not be considered separately when choosing the method for palatal expansion in adults. In these situations Surgically Assisted Rapid Maxillary Expansion (SARME) can be an option. And the choice of the surgical technique (SARME) should focus mainly the patient's age, grade of skeletal maturation, anatomical structures that offer more resistance to the maxillary expansion and location of the palatal constriction. Hyrax is the expander appliance more indicated for patients that will be submitted to SARME.
\end{abstract}

Keywords: Maxillary expansion. Adults. Transverse maxillary deficiency. 


\section{REFERÊNCIAS}

1. ANTTILA, A.; FINNE, K.; KESKE-NISULA, K. et al. Feasibility and long-term stability of surgically assisted rapid maxillary expansion with lateral osteotomy. Eur. J. Orthod., Oxford, v. 26, no. 4, p. 391-395, Aug. 2004.

2. BELL, W. H.; EPKER, B. N. Surgical-orthodontic expansion of the maxilla. Am. J. Orthod., St. Louis, v. 54, no. 5, p. 517-528, 1976.

3. BETTS, N. J.; VANARSDALL, R. L.; BARBER, H. D. et al. Diagnosis and treatment of transverse maxillary deficiency. Int. J. Adult Orthodon. Orthognath. Surg., Chicago, v. 10, no. 2, p. 75-96, 1995.

4. BIEDERMAN, W. A hygienic appliance for rapid expansion. JPO: J. Pract. Orthod., Hempstead, v. 2, no. 2, p. 1-21, 1968.

5. BYLOFF, F. K.; MOSSAZ, C. F. Skeletal and dental changes following surgically assisted rapid palatal expansion. Eur. J. Orthod., Oxford, v. 26, no. 4, p. 403-409, 2004

6. CAPELOZZA FILHO, L.; CARDOSO NETO, J.; SILVA FILHO, O. G.; URSI, W. J. Non-surgically assisted rapid maxillary expansion in adults. Int. J. Adult Orthodon. Orthognath. Surg., Chicago, v. 11, no. 1, p. 57-66, jan./fev. 1996.

7. FISH, L. C.; EPKER, B. N. Prevention of relapse in surgical orthodontic treatment. Part I. Mandibular procedures. J. Clin. Orthod., Boulder, v. 20, no. 12, p. 826-841, 1986.

8. GLASSMAN, A. S.; NAHIGIAN, S. J.; MEDWAY, J. M. et al. Conservative surgical adults rapid palatal expansion: Sixteen cases. Am. J. Orthod., St. Louis, v. 86, no. 3, p. 207-203, 1984.

9. HAAS, A. J. Entrevista. R. Dental Press Ortodon. Ortop. Facial, Maringá, v. 6, n. 1, p. 1-10, jan./fev. 2001

10. HAAS, A. J. Long-term posttreatment evaluation of rapid palatal expansion. Angle Orthod., Appleton, v. 50, no. 3, p. 189-217, 1980.

11. HAAS, A. J. Palatal expansion: Just the beginning of dentofacial orthopedics. Am. J. Orthod., St. Louis, v. 57, no. 3, p. 219-255, 1970.

12. HAAS, A. J. Rapid expansion of the maxillary dental arch and nasal cavity by opening the mid-palatal suture. Angle Orthod. Appleton, v. 31, no. 2, p. 73-90, 1961

13. HAAS, A. J. The treatment of maxillary deficiency by opening the midpalatal suture. Angle Orthod., Appleton, v. 35, no. 3, p. 200-217, 1965.

14. HANDELMAN, C. S. Nonsurgical rapid maxillary alveolar expansion in adults: A clinical evaluation. Angle Orthod., Appleton, v. 67, no. 4, p. 291-305, 1997.

15. HANDELMAN, C. S.; WANG, L.; BeGOLE, E. A.; HAAS, A. J. Nonsurgical rapid maxillary expansion in adults: Report on 47 cases using the Haas expander. Angle Orthod., Appleton, v. 70 , no. 2, p. 129-144, 2000.
16. ISAACSON, R. J.; INGRAM, A. H. Forces produced by rapid maxillary expansion - II. Forces present during treatment. Angle Orthod., Appleton, v. 34, no. 4, p. 261-270, 1964.

17. ISERI, H.; OZSOY, S. Semirapid maxillary expansion: A study of long-term transverse effects in older adolescents and adults. Angle Orthod., Appleton, v. 74, no. 1, p. 71-78, Feb. 2004.

18. JACOUBS, J. D.; BELL, W. H.; WILLIAMS, C. E. et al. Control of transverse dimension with surgery and orthodontics. Am. J. Orthod., St. Louis, v. 77, no. 3, p. 284-306, 1980.

19. LIMA, A. L.; SILVA FILHO, R. M. A.; BOLOGNESE, A. M. Longterm clinical outcome of rapid maxillary expansion as the only treatment performed in Class I malocclusion. Angle Orthod., Appleton, v. 75, no. 3, p. 416-420, 2005.

20. LINES, P. A. Adults rapid maxillary expansion with corticotomy. Am. J. Orthod., St. Louis, v. 67, no. 1, p. 44-56, 1975.

21. McNAMARA, J. A. Maxillary transverse deficiency. Am. J. Orthod. Dentofacial Orthop., St. Louis, v. 117, no. 5, p. 567-570, May 2000.

22. MEDEIROS, P. J.; MEDEIROS, P. P. Cirurgia ortognática para o ortodontista. 1. ed. São Paulo: Ed. Santos, 2001.

23. MELSEN, B. Palatal growth studied on human autopsy material. Am. J. Orthod., St. Louis, v. 68, no. 1, p. 42-54, 1975.

24. MOSSAZ, C. F.; BYLOFF, F. K.; RICHTER, M. Unilateral and bilateral corticotomies for correction of maxillary transverse discrepancies. Eur. J. Orthod., Oxford, v. 14, no. 2, p. 110-116, 1992.

25. NORTHWAY, W. M. Surgically assisted rapid maxillary expansion: A comparison of technique, response, and stability. Angle Orthod., Appleton, v. 67, no. 4, p. 309-320, 1997

26. PEARSON, M.; THILANDER, B. Palatal suture closure in man. Am. J. Orthod. St. Louis, v. 72, no. 1, p. 42-52, July 1977.

27. POGREL, M. A.; KABAN, L. B.; VARGERVIK, K.; BAUMRIND, S. Surgically assisted rapid maxillary expansion in adults. Int. J. Adult Orthodon. Orthognath. Surg., Chicago, v. 7, no. 1, p. 37-41, 1992

28. PROFFIT, W. R.; FIELDS, H. W. Ortodontia contemporânea. 2. ed. Rio de Janeiro: Guanabara Koogan, 1995.

29. REVELO, B.; FISHMAN, L. S. Maturational evaluation of ossification of the midpalatal suture. Am. J. Orthod.

Dentofacial Orthop., St. Louis, v. 105, no. 3, p. 288-292, 1994

30. TAVANO, O. A radiografia carpal como estimador da idade óssea: manual de ensino. Bauru: Faculdade de Odontologia de Bauru, 1994.

31. WERTZ, R. A. Skeletal and dental changes accompanying rapid midpalatal suture opening. Am. J. Orthod., St. Louis, v. 58, no. 1, p. 41-66, July 1970.
Endereço para correspondência

Rowdley Robert Pereira Rossi

Av. Hugo Viola, 1001 - loja 05, Jardim da Penha

CEP: 29.060-420 - Vitória / ES

E-mail: rowdley@yahoo.com.br 